Supporting information

\title{
Multilayer transfer printing on micro-reservoir-patterned substrate employing hydrophilic composite mold for selective immobilization of biomolecules
}

Nae Yoon Lee, Ju Ri Lim, Min Jung Lee, Sungsu Park, and Youn Sang Kim*

Division of Nano Sciences and Department of Chemistry, Ewha Womans University, 11-1 Daehyun-dong, Seodaemun-gu, Seoul, 120-750, Korea 
(a)

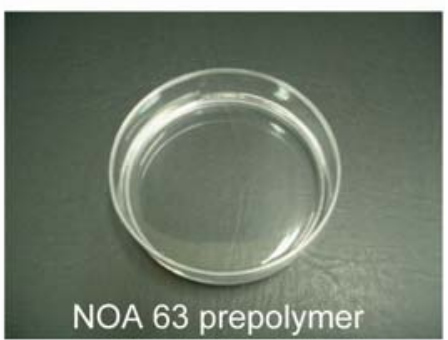

(b)

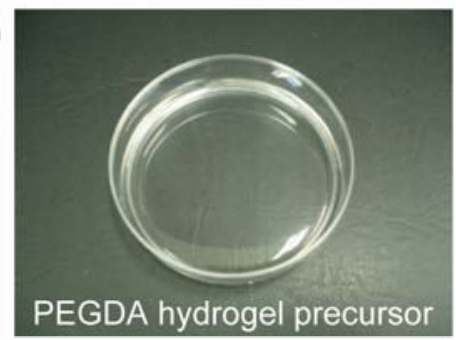

(c)

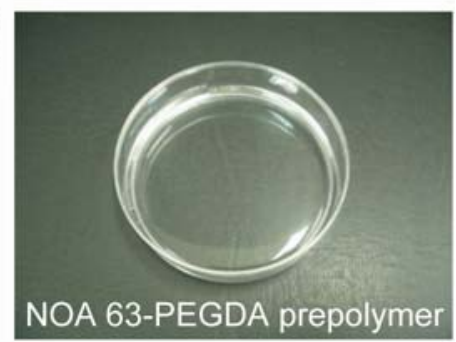

(d)

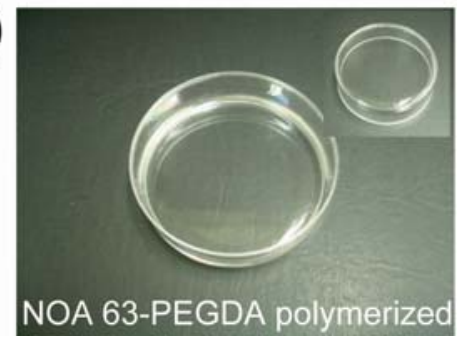

Figure S1. Photos taken before UV polymerization $(\lambda=365 \mathrm{~nm}$ ) for (a) NOA 63 prepolymer, (b) PEGDA hydrogel precursor solution containing photoinitiator, 2-hydroxy-2methylpropiophenone (HOMPP), and (c) homogeneous mixture of NOA 63 and PEGDA prepolymers in the weight ratio of 6:4. (d) Mixture of NOA 63 and PEGDA prepolymers after UV polymerization $(\lambda=365 \mathrm{~nm}$ ) for $30 \mathrm{~min}$. The inset is the photo of the Petri-dish in (d) put upside down to demonstrate that the mixture has been polymerized. 

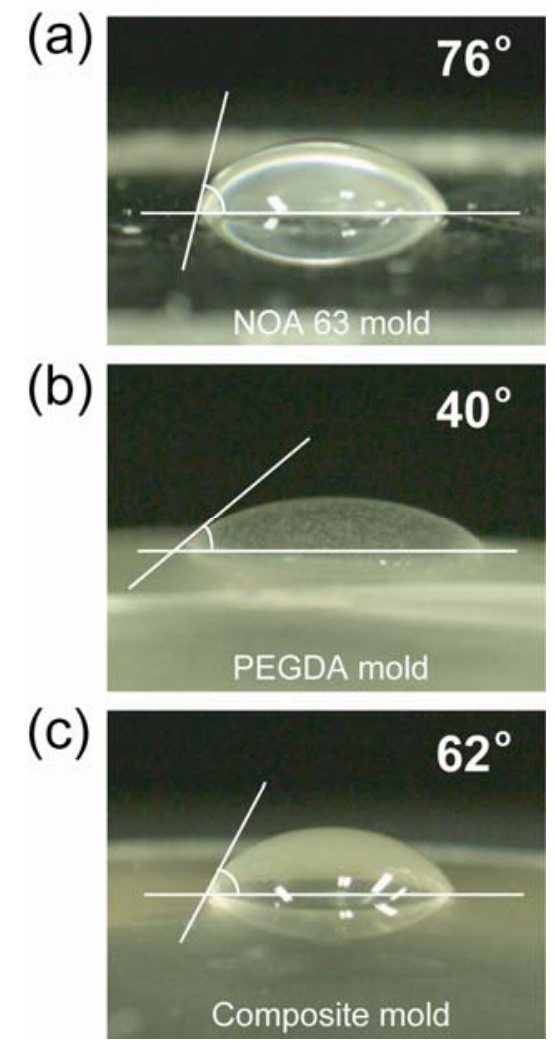

Figure S2. Advancing water contact angles measured on (a) NOA 63 mold, (b) PEGDA mold, and (c) 6:4 (w/w) NOA 63-PEGDA composite mold. 
(a)

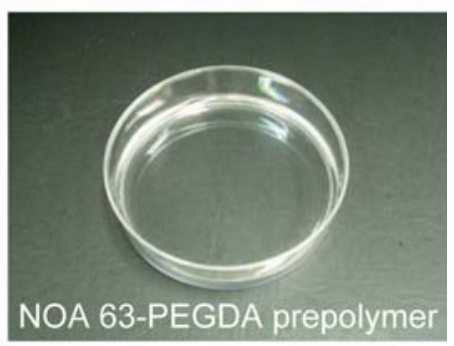

(b)

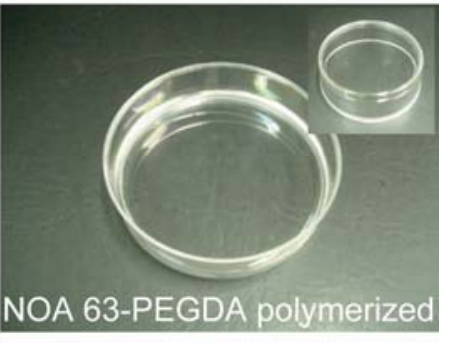

(c)

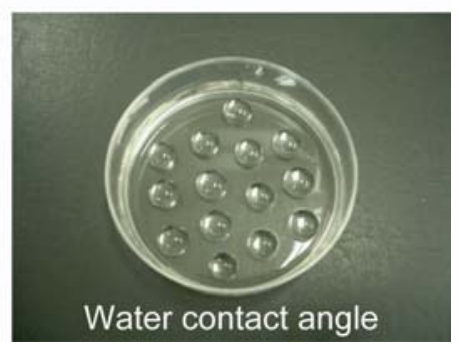

Figure S3. NOA 63-PEGDA prepolymer thoroughly blended and left undisturbed for $20 \mathrm{~h}$. (a) Before UV polymerization. (b) After UV polymerization. (c) Regional water wettability observed on the surface of the 6:4 (w/w) NOA63-PEGDA composite mold (volume of each water droplet $=50 \mu \mathrm{L}$ ). 